\title{
25 \\ Robustness margins and robustification of nominal explicit MPC
}

P. Rodríguez-Ayerbe, S. Olaru, A. Nguyen, R. Koduri

L2S, CentraleSupélec - CNRS - UPS

Université Paris-Saclay

29 June 2016

European Control Conference 


\section{Outline}

$>$ Problem statement

$>$ Robustness margins

- Polytopic uncertainty

- Gain margin

- Neglected dynamics

$>$ Robustification of explicit solutions 


\section{Problem statement}

$>$ A nominal discrete time linear systems

$$
\left\{\begin{array}{l}
x(k+1)=A x(k)+B u(k) \\
y(k)=C x(k)
\end{array}\right.
$$

$>$ A function $u_{p w a}: X \rightarrow \mathbb{R}^{p}$ defined over a polyhedral partition $X=\bigcup_{i \in I_{N}} X_{i} \subset \mathbb{R}^{n}$

$$
\begin{aligned}
& u_{p w a}(x)=G_{i} x+g_{i} \text { for } x \in X_{i} \\
& G_{i} \in \mathbb{R}^{p \times n} g_{i} \in \mathbb{R}^{p \times 1}
\end{aligned}
$$

Is a piecewise affine control law over the partition $X$ 


\section{Problem statement}

$>$ Polyhedral state partition

A polyhedral partition of a compact set $X \subset \mathbb{R}^{n}$ is defined as follows:

1) $X=\bigcup_{i \in I_{N}} X_{i}, N \in N_{+}$

2) $X_{i}$ is polyhedral $\forall i \in I_{N}$

3) $\operatorname{int}\left(X_{i}\right) \cap \operatorname{int}\left(X_{j}\right)=\varnothing$ with $i \neq j,(i, j) \in I_{N}^{2}$

4) $\left(X_{i}, X_{j}\right)$ are neighbours if $(i, j) \in I_{N}^{2}$,

$i \neq j$ and $\operatorname{dim}\left(X_{i} \cap X_{j}\right)=n-1$

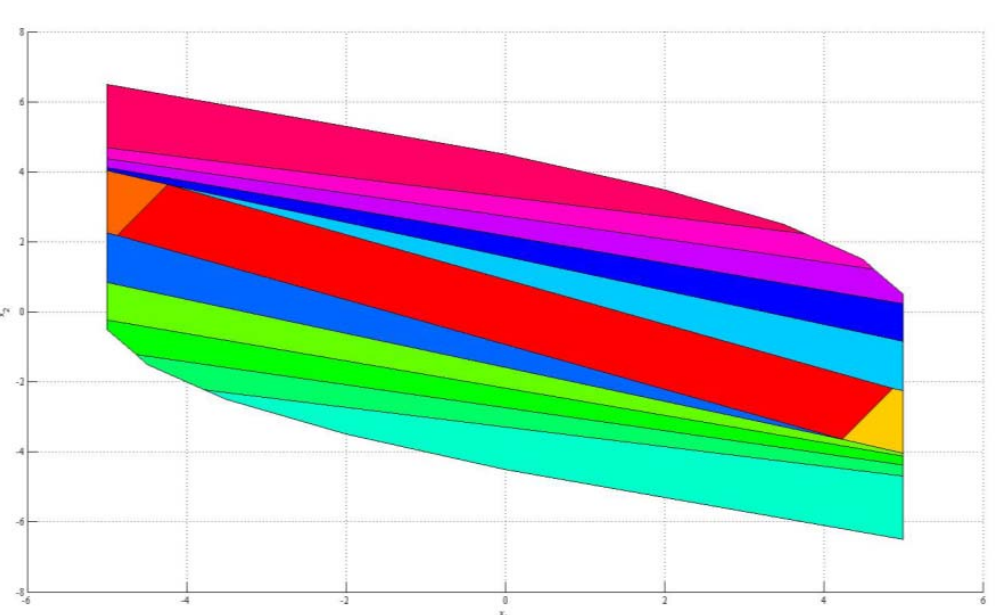




\section{Problem statement}

- Several popular techniques lead to such a problem formulation, among which we can mention for example the Model Predictive Control in its explicit form (Bemporad et al, Seron et al, Tondel et al., Olaru and Dumur).

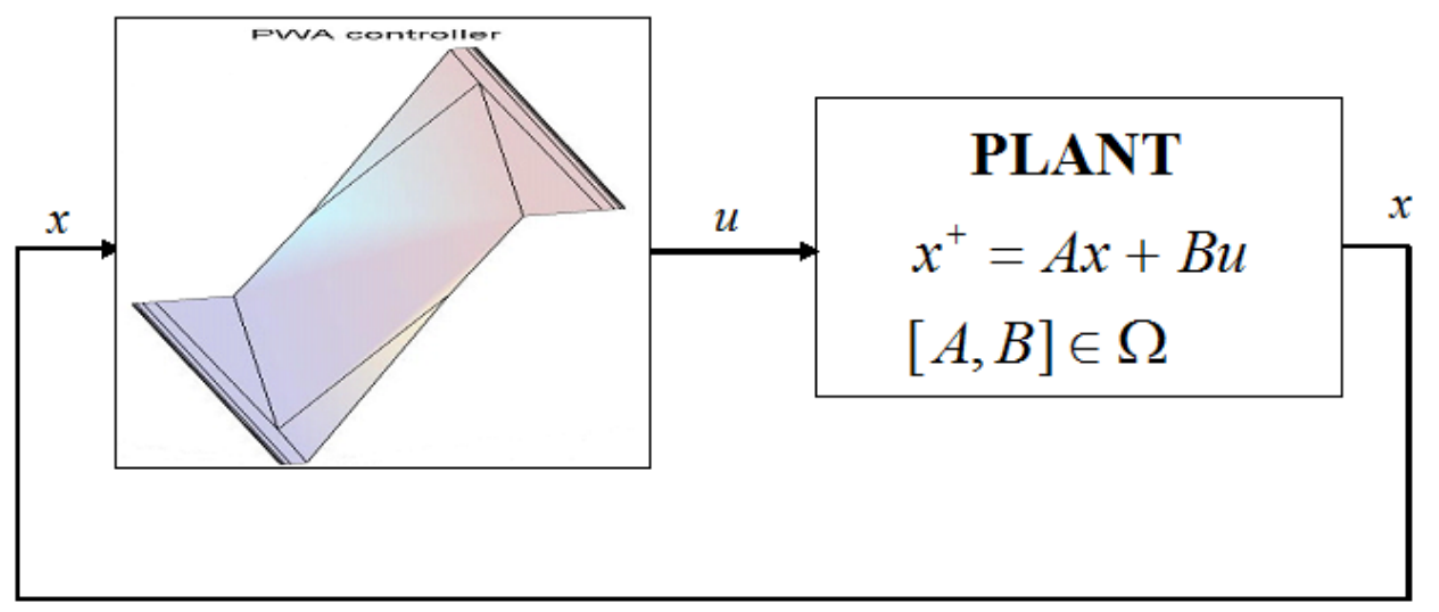




\section{Problem statement}

\section{$>$ Goals:}

- To analyse the inherent robustness of PWA control towards model uncertainties $\rightarrow$ robustness margin problems.

- To further tune the original controller to improve robustness margin.

- Synthesize explicit solution with good robustness margin. 


\section{Outline}

$>$ Problem statement

$>$ Robustness margins

- Polytopic uncertainty

- Gain margin

- Neglected dynamics

$>$ Robustification of explicit solutions 


\section{Robustness margins}

- Nominal closed loop

The nominal closed loop dynamics represent a PWA system:

$$
x(k+1)=f_{p w a}(x(k))=\left(A_{0}+B_{0} G_{i}\right) x(k)+B_{0} g_{i} \text { for } x(k) \in X_{i}
$$

- Positive invariance

$\Rightarrow$ A set $X \in \mathbb{R}^{n}$ is posive invariant with respect to the system $x(k+1)=f_{p w a}(x(k), w)$ if for any $x\left(k_{0}\right) \in X$, $x(k) \in X \quad \forall k>\mathbb{N}$
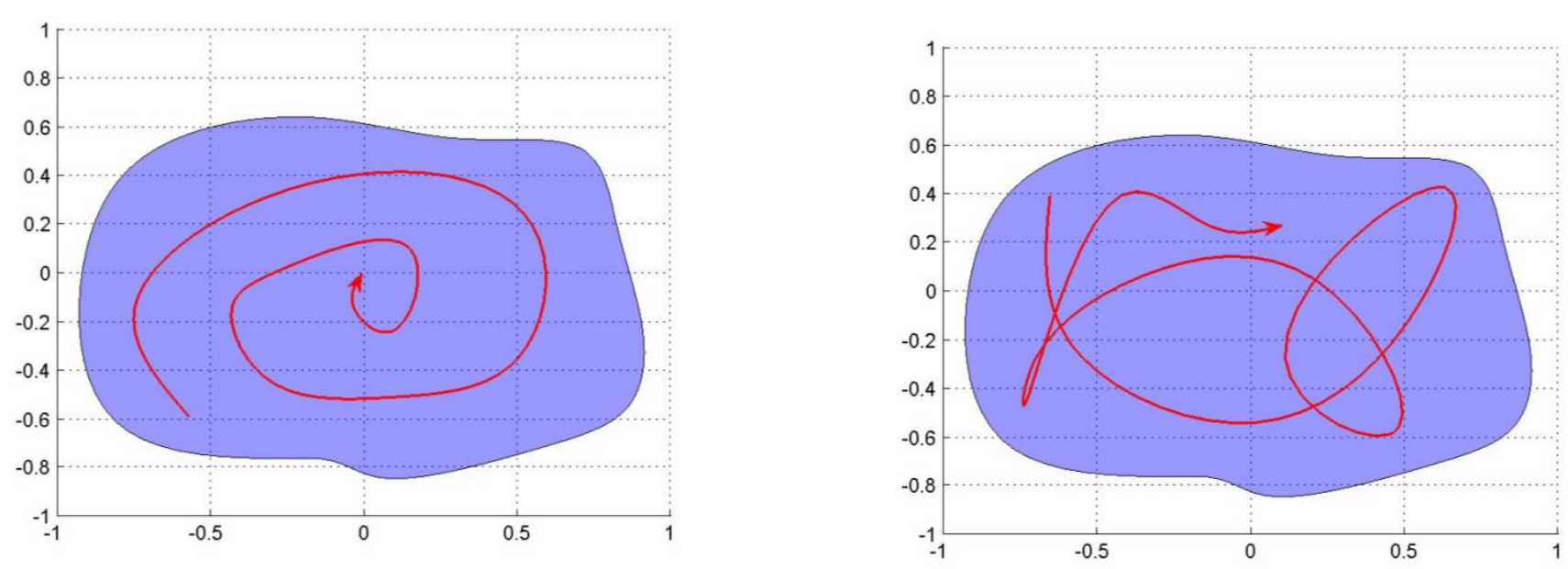


\section{Robustness margins}

- Assumptions

- The set $X$ is a polytope

- The set $X$ is positive invariant with respect to the nominal model

- The control $u_{p w a}: \mathbb{R}^{n} \rightarrow \mathbb{R}^{m}$ is continuous

- The origin is the only fixed point of the nominal dynamics

- The origin is asymptotically stable with $X$ as basin of attraction 


\section{Robustness margins}

- Robust invariance for polytopic uncertainty

$$
\begin{aligned}
& \left\{\begin{array}{l}
x(k+1)=A(k) x(k)+B(k) u(k) \\
y(k)=C x(k)
\end{array}\right. \\
& {[A(k) B(k)] \in \Psi=\operatorname{conv}\left(\left[\mathrm{A}_{1}, \mathrm{~B}_{1}\right], \ldots\left[\mathrm{A}_{L}, \mathrm{~B}_{L}\right]\right)}
\end{aligned}
$$
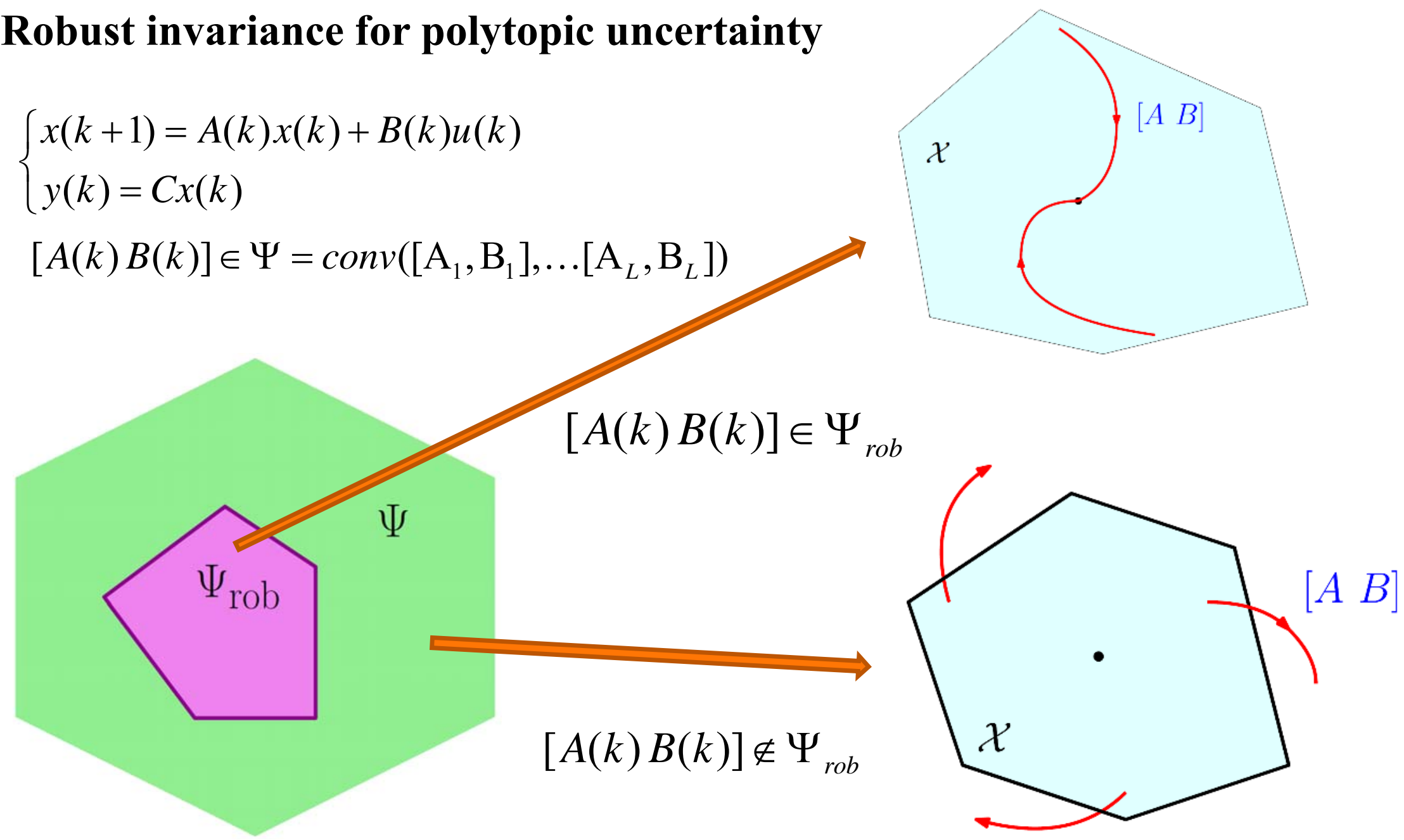


\section{Robustness margins}

- Robust invariance for polytopic uncertainty

$\Rightarrow \Psi_{\text {rob }}$ is polytopic
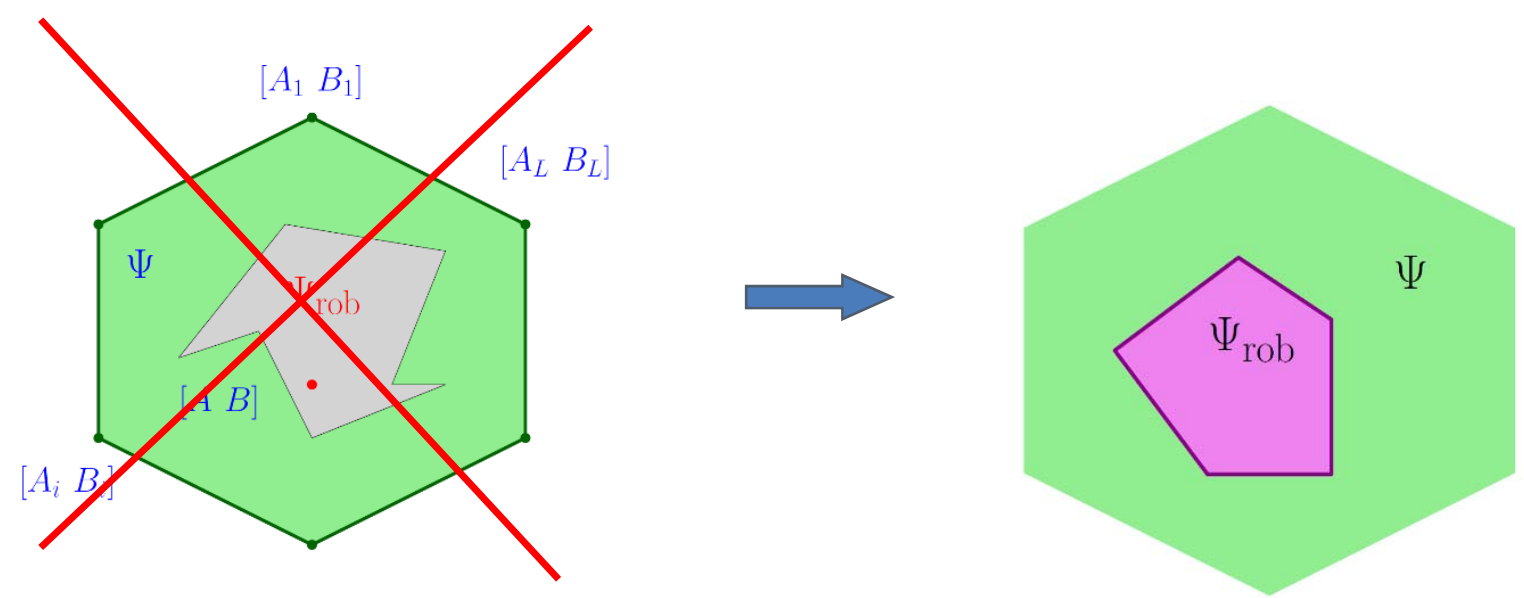

$$
\Psi_{\text {rob }}^{\alpha}=\left\{\alpha \in \mathbb{R}_{+}^{L} \mid \sum_{j=1}^{L} \alpha_{j}\left(A_{j}+B_{j} G_{i}\right) X_{i} \oplus \alpha_{j} B_{j} g_{i} \subset X, \forall_{i} \in I_{N}, \mathbf{1}^{T} \alpha=1\right\}
$$

$\Rightarrow \Psi_{\text {rob }}$ can be computed using vertex or half-space representation of

$$
X=\bigcup_{i \in I_{N}} X_{i}, N \in N_{+}
$$




\section{Robustness margins}

- For half-space representation:

$$
\begin{gathered}
X=\left\{x \in \mathbb{R}^{n} \mid F x \leq h\right\} \quad X_{i}=\left\{x \in \mathbb{R}^{n} \mid F_{i} x \leq h_{i}\right\} \\
\Psi_{\text {rob }}^{\alpha}=\operatorname{Proj}_{\mathbb{R}^{\mathrm{L}}} P \\
\left.P=\left\{\left(\alpha, M_{1}, \ldots M_{N}\right) \mid \begin{array}{l}
\sum_{j=1}^{L} \alpha_{j} F\left(A_{j}+B_{j} G_{i}\right)=M_{i} F_{i}, \\
M_{i} h_{i} \leq h-F \sum_{j=1}^{L} \alpha_{j} B_{j} g_{i},
\end{array}\right\} i \in I_{N}\right\}
\end{gathered}
$$




\section{Robustness margins}

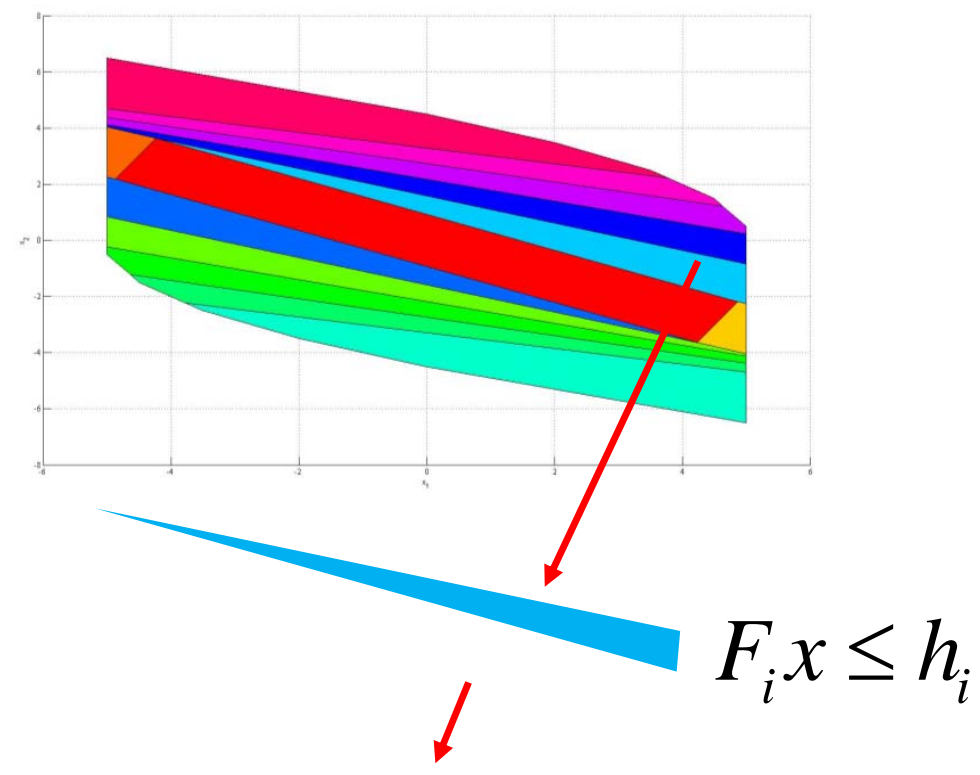

$$
P_{2}=\{x \mid F x \leq h\}
$$

$P_{1}=\left\{x \mid F_{i}\left(A(k)+B(k)\left(G_{i} x+g_{i}\right)\right) \leq h_{i}\right\}$

$P_{1} \subseteq P_{2} \Leftrightarrow \exists M$ s.t.

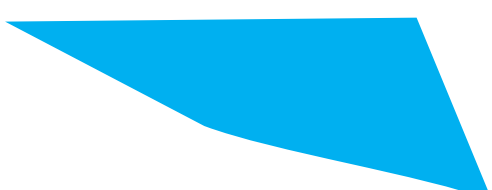

$\Longrightarrow$ Positive Invariance if : $P_{1} \subseteq P_{2}$

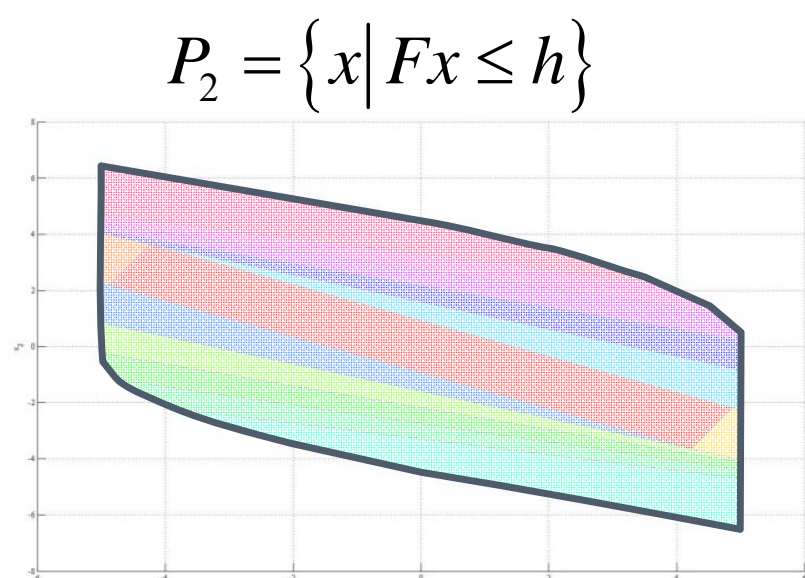

$$
\left\{\begin{array}{l}
\operatorname{vec}(M) \geq 0, \\
M F_{1}=F_{2} \\
M h_{1} \leq h_{2}
\end{array}\right.
$$




\section{Robustness margins}

- Numerical example

$$
\begin{aligned}
& A_{1}=\left[\begin{array}{cc}
1 & 1 \\
0.9 & 0.5
\end{array}\right] A_{2}=\left[\begin{array}{cc}
2 & 1 \\
1.5 & 1.5
\end{array}\right] A_{3}=\left[\begin{array}{ll}
1.5 & 1 \\
3.8 & 1
\end{array}\right] \\
& B_{1}=B_{2}=B_{3}=\left[\begin{array}{l}
1 \\
1
\end{array}\right]
\end{aligned}
$$

In presence of constraints on the control variable and the output variable

$$
\begin{aligned}
& -0.2 \leq u_{k} \leq 0.2 \\
& -0.5 \leq y_{k} \leq 0.5
\end{aligned}
$$

With the nominal model chosen to synthesize a PWA controller law

$$
\begin{aligned}
& A_{0}=0.5 A_{1}+0.3 A_{2}+0.2 A_{3} \\
& B_{0}=B_{1}
\end{aligned}
$$




\section{Robustness margins}

- Numerical example

The partition of the obtained PWA control law

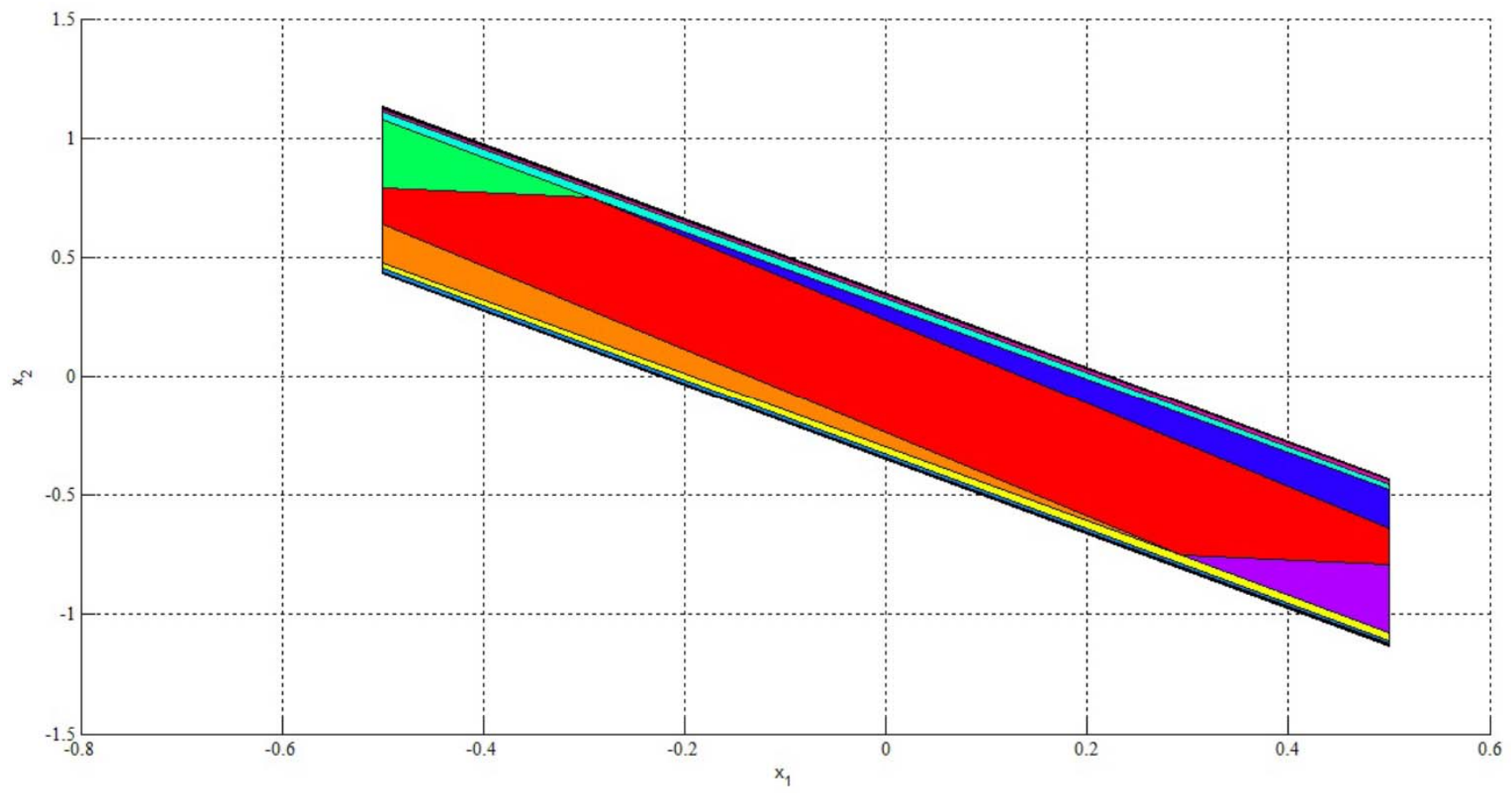




\section{Robustness margins}

- Numerical example

Irob projected on the plane $\left[\alpha_{1}, \alpha_{2}\right]$. Note that $\alpha_{3}=1-\alpha_{1}-\alpha_{2}$

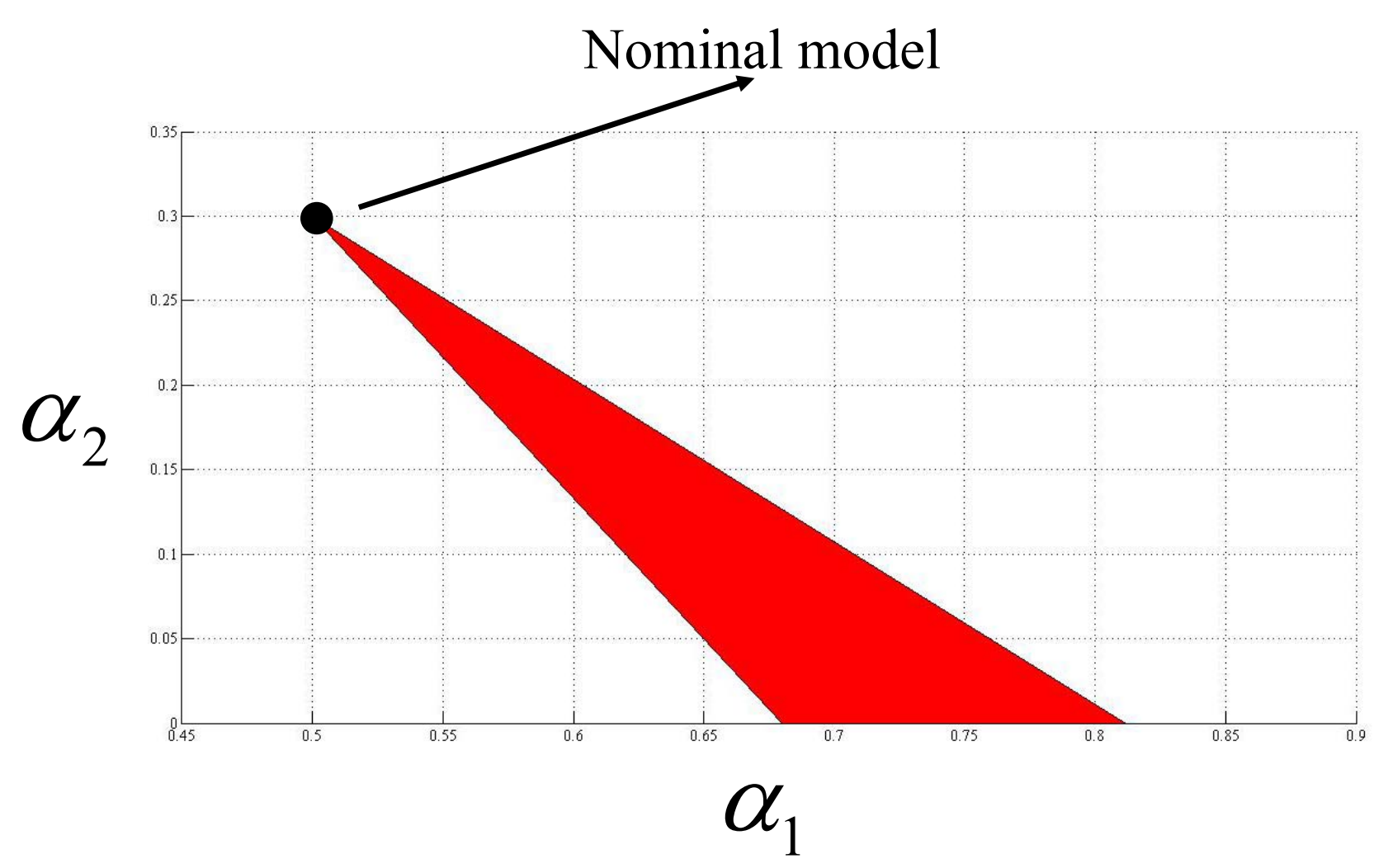




\section{Robustness margins}

- Gain margin

The gain margin is represented by the set $K \subset \mathbb{R}^{m}$, such that:

$$
x(k+1)=A x(k)+B\left(I_{m}+\operatorname{diag}\left(\delta_{K}\right)\right) u_{p w a}(x(k)) \in X, \forall x(k) \in X \text { and } \delta_{K} \in K
$$

$K=\bigcap_{q=1}^{p} K_{q}$

$K_{q}=\left\{z \in \mathbb{R}^{m} \mid \exists u \in \Delta U_{q}, M z=u\right\}$

$$
\begin{aligned}
& \Delta U_{q}=\operatorname{Proj}_{U} H_{q} \\
& H_{q}=\left\{(\delta u, \lambda) \in \mathbb{R}^{m} \times \mathbb{R}^{r} \text {, and }[A B]\left[\begin{array}{c}
x(k) \\
u_{p w a}(x(k))
\end{array}\right]+B \delta u=W \lambda\right\}
\end{aligned}
$$

$W$ stores the vertices of $X: \quad W \in \mathbb{R}^{n \times r}, W=\left[w_{1}, \ldots w_{r}\right]$ 


\section{Robustness margins}

- Gain margin: numerical example

Consider a linear discrete system with two inputs and two outputs

$$
\begin{aligned}
x(k+1) & =\left[\begin{array}{ccc}
1.2 & -1.0 & 0 \\
0 & -1.2 & 0.5 \\
0.2 & 0.4 & 0
\end{array}\right] x(k)+\left[\begin{array}{cc}
1.0 & 0.2 \\
0.5 & 0 \\
0 & 0.7
\end{array}\right] u(k) \\
y(k) & =\left[\begin{array}{lll}
1 & 0 & 0 \\
0 & 0 & 1
\end{array}\right] x(k) .
\end{aligned}
$$

The weight applied on the control inputs and state vectors are

$$
Q=\left[\begin{array}{lll}
5 & 0 & 0 \\
0 & 1 & 0 \\
0 & 0 & 1
\end{array}\right] \text { and } R=\left[\begin{array}{cc}
0.5 & 0 \\
0 & 1
\end{array}\right]
$$

The input constraints, $\left[\begin{array}{l}-2 \\ -2\end{array}\right] \leq u(k) \leq\left[\begin{array}{l}2 \\ 2\end{array}\right]$ and the output

constraints $\left[\begin{array}{l}-2 \\ -2\end{array}\right] \leq y(k) \leq\left[\begin{array}{l}2 \\ 2\end{array}\right]$. Prediction horizon $N_{p}=2$ 


\section{Robustness margins}

- Gain margin: numerical example

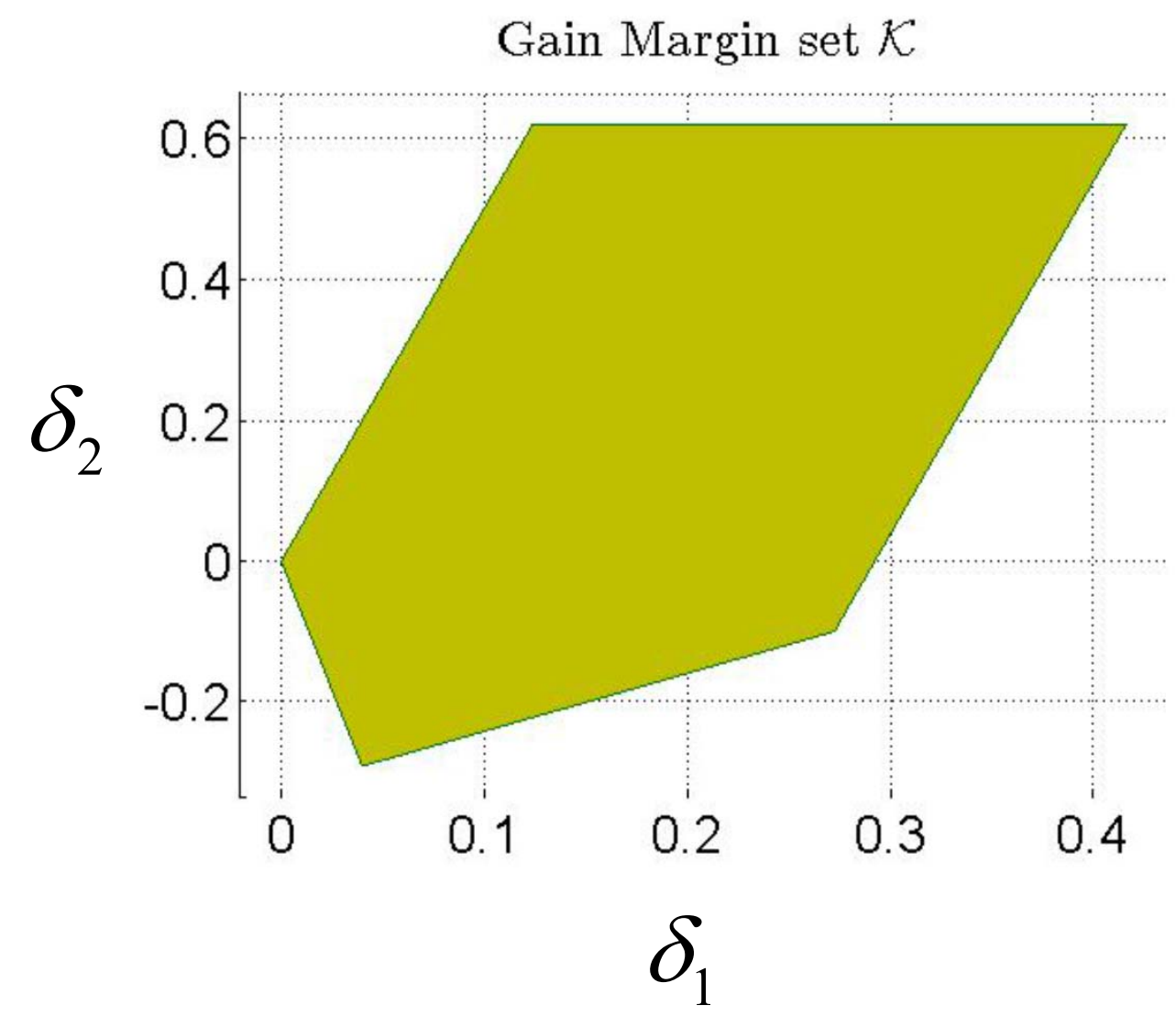




\section{Robustness margins}

- Neglected dynamics

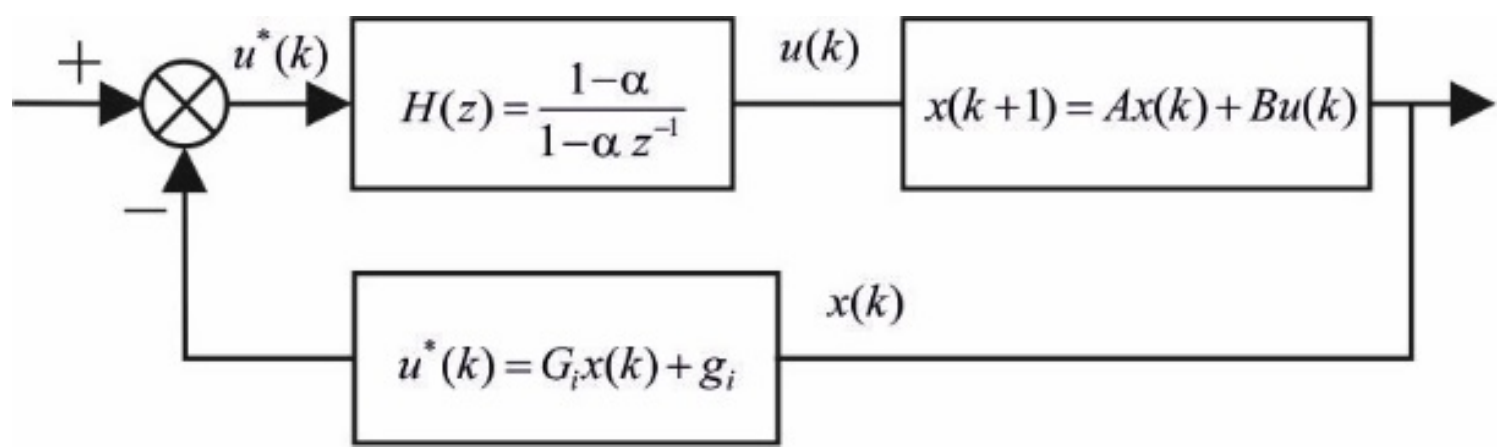

The augmented model is:

$$
\begin{aligned}
& x_{e}(k+1)=\left[\begin{array}{cc}
A & \alpha B \\
0 & \alpha
\end{array}\right] x_{e}(k)+\left[\begin{array}{c}
B(1-\alpha) \\
\alpha
\end{array}\right] u(k) \\
& u(k)=\left(x_{e}(k)\right)=\left[\begin{array}{ll}
G_{i} & 0
\end{array}\right] x_{e}(k)+g_{i} \text { for } x(k) \in X_{i}
\end{aligned}
$$




\section{Robustness margins}

- Neglected dynamics

$$
x_{e}(k+1)=\left[\begin{array}{cc}
A & \alpha B \\
0 & \alpha
\end{array}\right] x_{e}(k)+\left[\begin{array}{c}
B(1-\alpha) \\
\alpha
\end{array}\right] u(k)
$$

Polytopic description of extended model

$$
\begin{gathered}
A_{1}=\left[\begin{array}{ll}
A & 0 \\
0 & 0
\end{array}\right] B_{1}=\left[\begin{array}{l}
B \\
1
\end{array}\right] A_{2}=\left[\begin{array}{cc}
A & B \\
0 & 1
\end{array}\right] B_{1}=\left[\begin{array}{l}
0 \\
0
\end{array}\right] \\
{\left[A_{e} B_{e}\right]=(1-\alpha)\left[A_{1} B_{1}\right]+\alpha\left[A_{2} B_{2}\right]} \\
\Omega^{\alpha}=\operatorname{Proj}_{\alpha} T \\
T=\left\{(\alpha, \Gamma) \in \mathbb{R} \times \mathbb{R}^{r \times p}(1-\alpha)\left(A_{1} V_{e}+B_{1} U_{e}\right)+\alpha\left(A_{2} V_{e}+B_{2} U_{e}\right)=W \Gamma\right\}
\end{gathered}
$$

$W$ stores the vertices of $X, V_{e}$ stores de vertices of $X_{i}$ : 


\section{Robustness margins}

- Neglected dynamics : numerical example

Consider the discrete time system given in the previous section. The margin for the first order neglected dynamics is represented by the set $\Omega^{\alpha}=\{0,0.195\}$. 


\section{L2S}

\section{Outline}

$>$ Problem statement

$>$ Robustness margins

- Polytopic uncertainty

- Gaim margin

- Neglected dynamics

$>$ Robustification of explicit solutions 


\section{Robustification of explicit solutions}

\section{Robustification of linear control law}

\section{Youla-Kučera parameterisation}

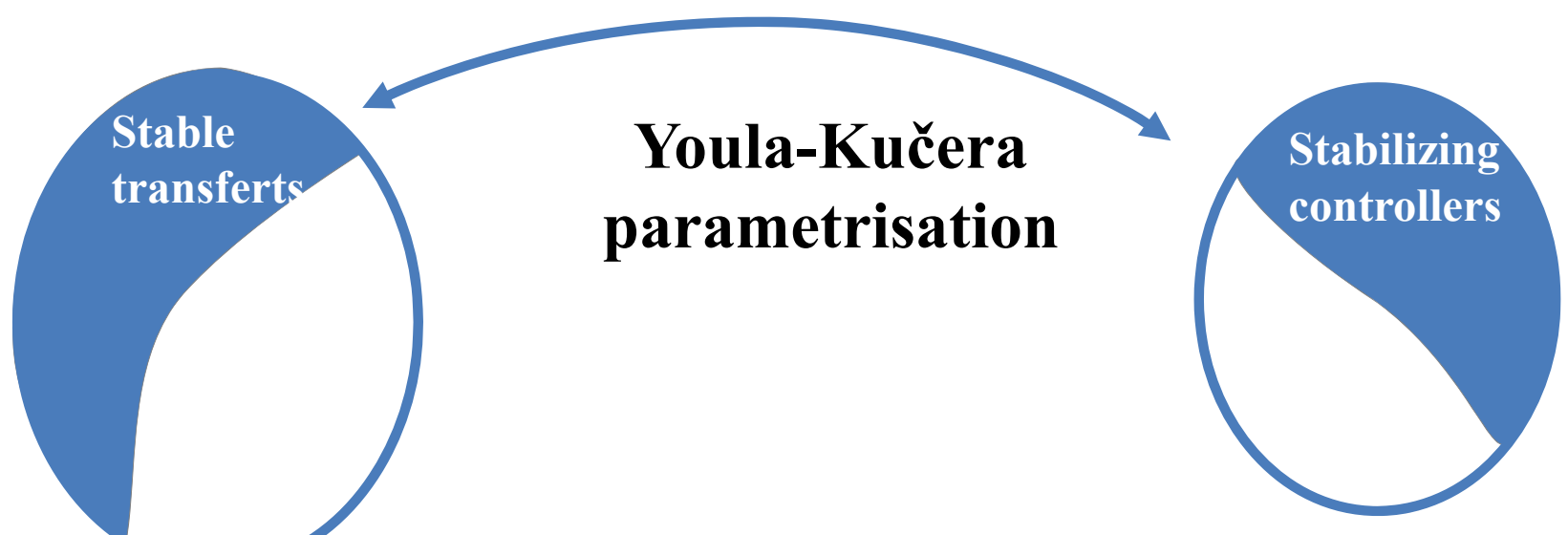

Linear time invariant transfers set
Linear time invariant transfers set 


\section{Robustification of explicit solutions \\ Youla-Kučera parameterisation}

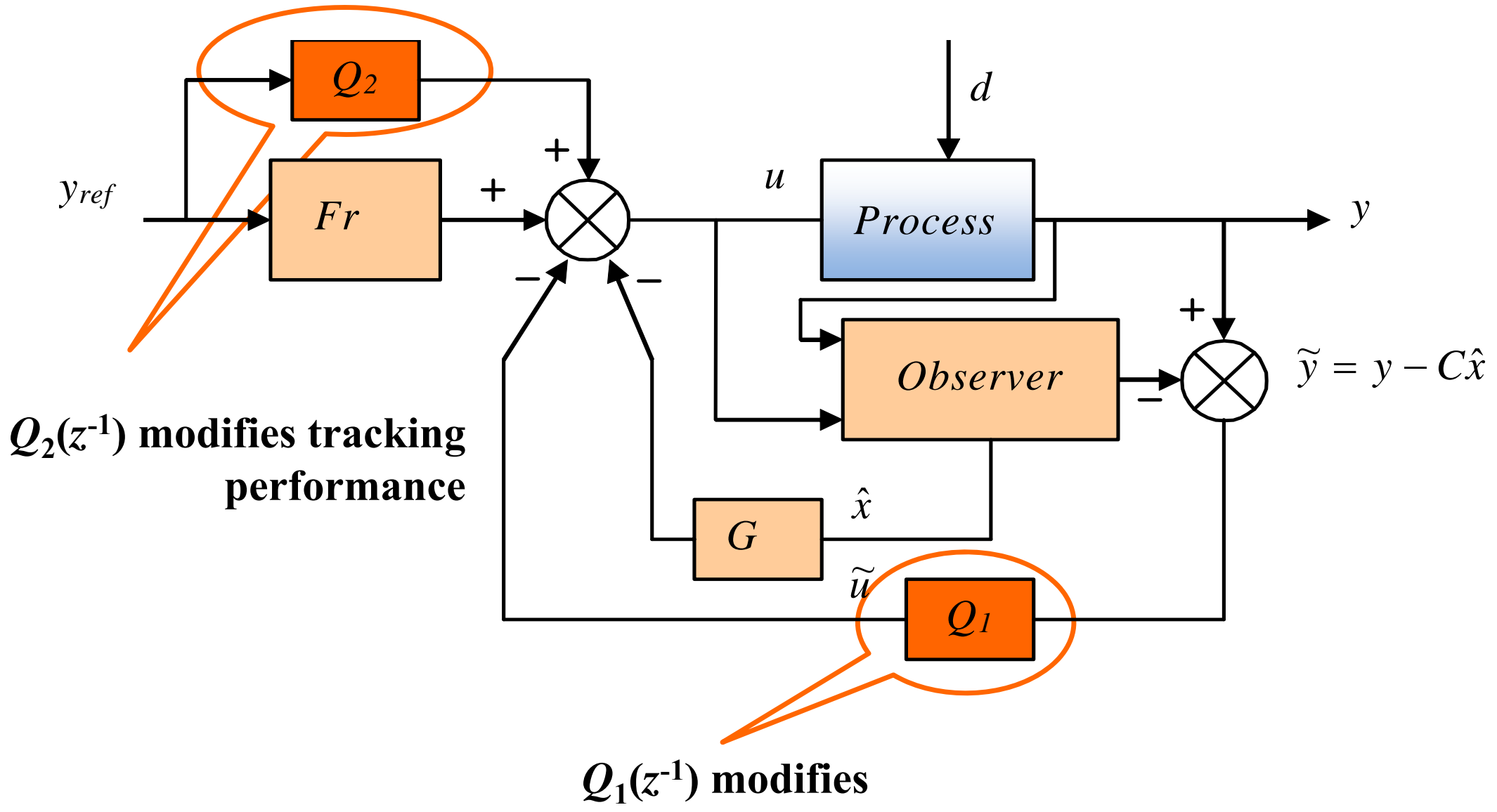

regulation dynamics 


\section{Robustification of explicit solutions}

- Youla-Kučera parametrisation can not be directly used to robustify a PWA controller because the continuity of the control law is lost

- The following approach is follow:

1. Robustification of unconstrained controller

2. Find the perturbance/noise model associated to the obtained controller

3. Use the new model to modify or the initial PWA controller

- This modification conserves the initial tracking behaviour

- The number of partitions is kept, as depends on the constraints 


\section{Robustification of explicit solutions}

Initial model

$$
\begin{array}{ll}
x_{t+1}=A x_{t}+B u_{t}+K e_{t} & \text { With } e_{t} \text { white } \\
y_{t}=C x_{t}+e_{t} & \text { noise }
\end{array}
$$

Cost function minimization without constraints

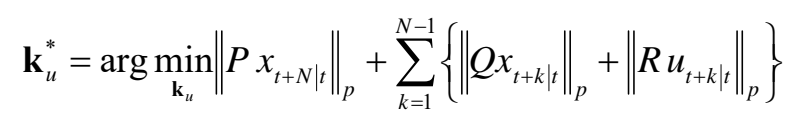

$\hat{x}_{t+1}=(A-K C-B L) \hat{x}_{t}+K y_{t} \quad$ Initial unconstrained $u_{t}=-L \hat{x}_{t}$ controller

\section{Q Parameter}

Robustified controller

$$
\left\{\begin{array}{l}
{\left[\begin{array}{c}
\hat{x}_{t+1} \\
X_{Q_{t+1}}
\end{array}\right]=\left[\begin{array}{cc}
A-K C-B L-B D_{Q} C & -B C_{Q} \\
-B_{Q} C & A_{Q}
\end{array}\right]\left[\begin{array}{c}
\hat{x}_{t} \\
X_{Q_{t}}
\end{array}\right]+\left[\begin{array}{c}
K-B D_{Q} \\
B_{Q}
\end{array}\right] y_{t}} \\
u_{t}=\left[-L+D_{Q} C-C_{Q}\right]\left[\begin{array}{c}
\hat{x}_{t} \\
X_{Q_{t}}
\end{array}\right]-D_{Q} y_{t}
\end{array}\right.
$$

Augmented model

$$
\begin{aligned}
& \underbrace{\left[\begin{array}{c}
x_{t+1} \\
x_{v+1}
\end{array}\right]}_{x_{t+t+1}}=\underbrace{\left[\begin{array}{cc}
A & K C_{v} \\
0 & A_{v}
\end{array}\right]}_{A_{t}}\left[\begin{array}{c}
x_{t} \\
x_{v t+1}
\end{array}\right]+\underbrace{\left[\begin{array}{c}
B \\
0
\end{array}\right]}_{B_{e}} \underbrace{u_{t}+}_{K_{e}}\left[\begin{array}{c}
K \\
B_{v}
\end{array}\right]_{t}^{e_{t}^{\prime}} \begin{array}{l}
\text { With } e_{t}^{\prime} \text { white } \\
\text { noise }
\end{array}
\end{aligned}
$$

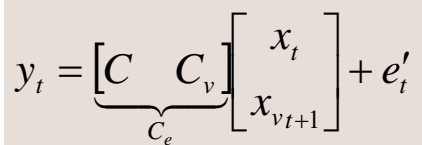

Cost function minimization without constraints

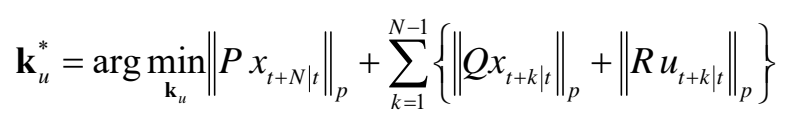

Unconstrained controller

$$
\begin{aligned}
& \hat{x}_{e t+1}=\left(A_{e}-K_{e} C_{e}-B_{e} L_{e}\right) \hat{x}_{e t}+K_{e} y_{t} \\
& u_{t}=-L_{e} \hat{x}_{e t}
\end{aligned}
$$




\section{Robustification of explicit solutions}

Two controllers are equivalents if the following equations are verified :

$$
\begin{aligned}
& D_{Q}-L_{e} K_{2}=0 \\
& A_{Q}-A_{v}+B_{v} C_{v}=0 \\
& C_{Q}-L_{v}+D_{Q} C_{v}=0 \\
& L_{v}=H^{-1} F_{v}\left(A_{v}, C_{v}\right)
\end{aligned}
$$

Non-linear equations :

- $F_{v}\left(A_{v}, C_{v}\right)$ depends on cost function

- Optimisation techniques can be used to solve the non-linear equation system

- The existence of the solution haven't been proved yet 


\section{Robustification of explicit solutions}

\section{Buck converter example}

Unconstrained controller robustification towards additive disturbances

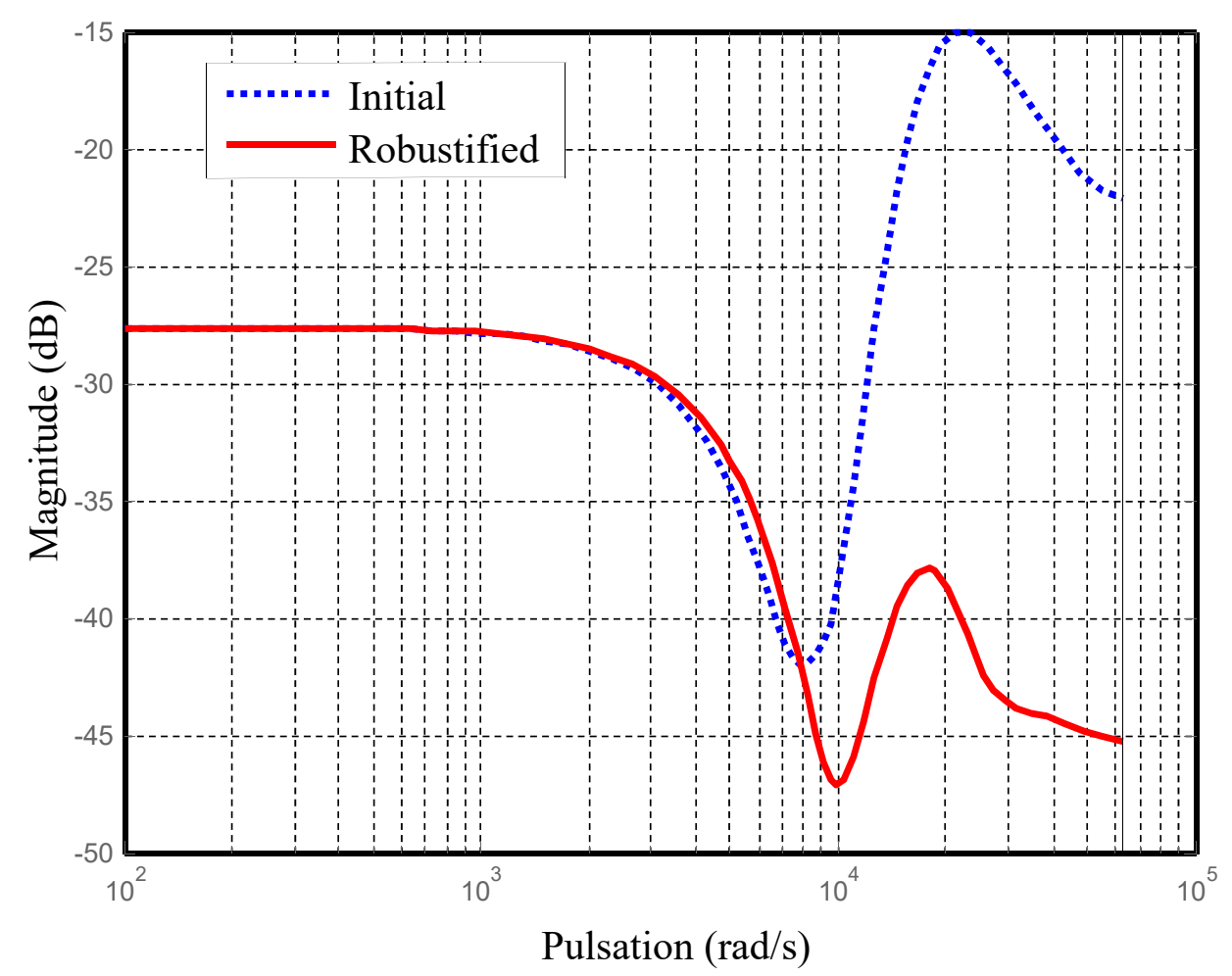




\section{Robustification of explicit solutions}

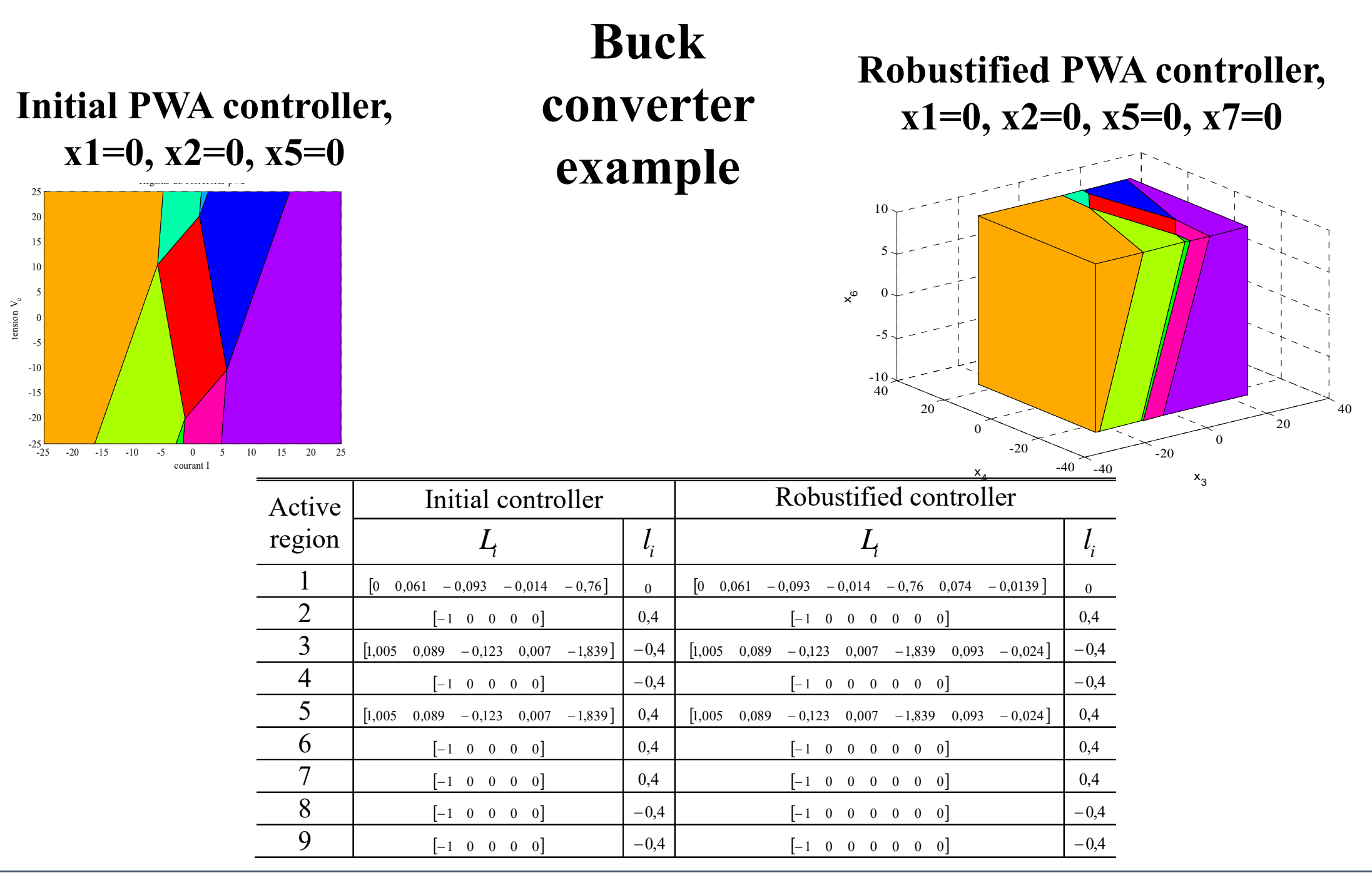




\section{Robustification of explicit solutions \\ Buck converter example}

\section{Output voltage step response}
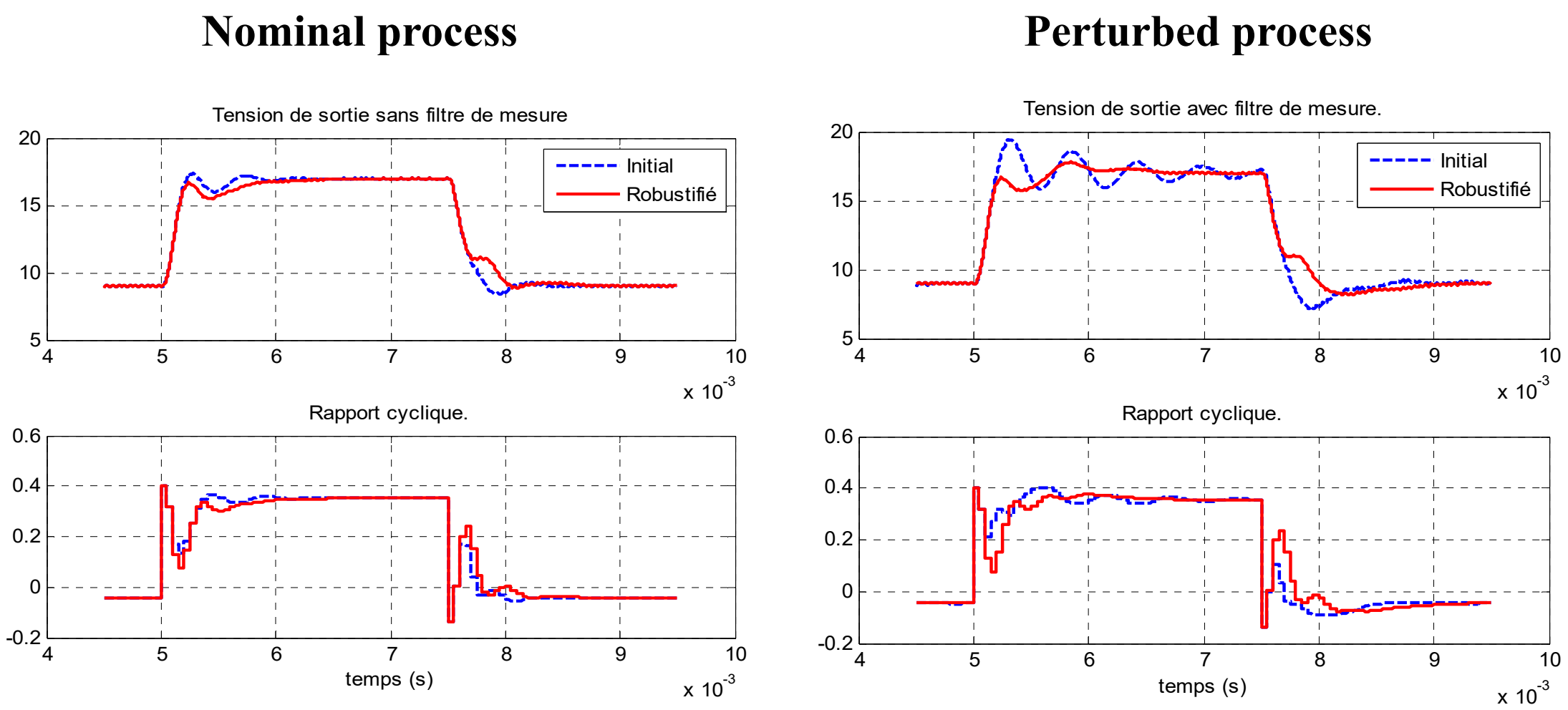


\section{References}

N. A. Nguyen, S. Olaru, P. Rodriguez-Ayerbe, "Explicit robustness and fragility margins for linear discrete systems with piecewise affine control law", Automatica, 2016, Vol. 68, pp. 334-343.

P. Rodriguez-Ayerbe and S. Olaru, "On the disturbance model in the robustification of explicit predictive control", International Journal of Systems Science, 2013, Vol. 44(5), pp. 853-864. 